\title{
Canon ambiental por vertidos en Costa Rica como instrumento económico en la gestión de aguas residuales: un enfoque metodológico y de análisis de la situación actual.
}

\author{
Rolando Sánchez-Gutiérrez ${ }^{1}$. \\ Wendy Villalobos-González². \\ 1. Universidad Nacional de Costa Rica. Investigador y académico. Laboratorio de Manejo del \\ Recurso Hídrico. Correo: rolando.sanchez.gutierrez@una.cr . Heredia, Costa Rica. \\ 2. Universidad Estatal a Distancia, Costa Rica. Encargada Cátedra de Ciencias Químicas. \\ Unidad de Investigaciones Bioenergéticas y Ambientales. Correo: \\ wvillalobosg@uned.ac.cr.Sabanilla, Costa Rica.
}

\section{RESUMEN}

Las aguas residuales constituyen una problemática mundial que atenta contra la salud de los ecosistemas hídricos y no es ajena para el estado costarricense. El manejo de aguas residuales en Costa Rica presenta un serio rezago, a pesar de esto, para el año 2017, existían 1769 puntos de vertido de agua residual aprobados por el estado. El reglamento del Cannon Ambiental por Vertidos (CAV) es un instrumento económico para la gestión de aguas residuales, que en principio, realiza una valoración económica de los daños ambientales ocasionados por los entes generadores al verter sus efluentes en los cuerpos de agua superficial, internalizando las externalidades negativas que esta actividad genera. Esta investigación presenta un análisis del diseño de la metodología empleada para la fijación de los montos de cobro en el CAV. Se determinó que desafortunadamente hoy en día, el cobro del CAV solo se realiza en función de los servicios ambientales prestados por los cuerpos receptores como sumidero de la carga contaminante (factores de descontaminación); existiendo una deficiencia en la estimación económica del canon, ya que no se considera la estimación económica de otros valores de usos y no usos del recurso natural. Además, se determinó que estos montos son relativamente bajos cuando se comparan contra las disposiciones de pago en otros países, haciendo aún más evidente la necesidad de incluir aquellas funciones ambientales afectadas y no contempladas en la metodología con la que se fijó el canon.

Palabras claves: reglamento, agua residual, canon, vertido, cuerpo receptor.

\section{ABSTRACT}

Environmental canon for discharges in Costa Rica as an economic tool for the wastewater management: A methodological approach and analysis of the current situation.

Wastewater is a world problem that threatens the health of water ecosystems and is no stranger to the Costa Rican state. The management of wastewater in Costa Rica presents a serious lag, despite this, for the year 2017, there were 1769 wastewater discharge points approved by the state. The regulation of the Environmental Cannon for Discharges (CAV) is an economic instrument for the management of wastewater, which in principle, performs an economic assessment of the environmental damage caused by the generating entities by pouring their effluents into the bodies of surface water, internalizing the negative externalities that this activity generates. This research presents an analysis of the design of the methodology used to set the collection amounts in the CAV. It was determined that unfortunately today, the collection of the CAV is only carried out based on the environmental services provided by the receiving bodies as a sink for the polluting load (decontamination factors); there is a deficiency in the economic estimate of the canon, since the economic estimate of other values of uses and nonuses of the natural resource is not considered. Furthermore, it was determined that these amounts are relatively low when compared against payment provisions in other countries, making even more evident the need to include those environmental functions affected and not contemplated in the methodology with which the royalty was established.

Key words: regulation, water, canon, discharge, receiving body. 


\section{Introducción}

El recurso hídrico es de suma importancia para el bienestar del ser humano y las comunidades, existe una estrecha relación entre el desarrollo económico y social de una zona y la calidad del recurso hídrico que la rodea (Onestini, 2011; Bower, 2014). Los ríos, por ejemplo, cumplen una función importante para la salud y el desarrollo económico del ser humano, representan una fuente importante de agua para consumo y uso en actividades productivas o de servicios (Massoud, 2012; Ha et al, 2017); además son parte del patrimonio cultural y natural, constituyendo ecosistemas con gran riqueza de biodiversidad. Durante las últimas décadas los depósitos más importantes de abastecimiento de agua, como acuíferos y ríos, han experimentado una alteración importante por diversos factores de origen antropogénico, entre ellos, los escasos valores éticos y ambientales de los usuarios, la falta de control y monitoreo por parte de las autoridades gubernamentales, además del vertido de aguas residuales con poco o nulo tratamiento (Barrenha et al., 2018; da Rocha et al., 2018).

El saneamiento de aguas residuales en Costa Rica presenta un serio rezago, principalmente por razones políticas, técnicas, financieras y culturales; a nivel estatal existe un traslape de funciones y responsabilidades de las instituciones involucradas propiciando un difícil escenario de gobernabilidad del agua (Ballestero y Reyes, 2006; Herrera-Murillo, 2017; MINAE, 2017), además existen pocos recursos destinados a la inversión en el fortalecimiento de infraestructura para el saneamiento (Ballestero y Reyes, 2006; Bower, 2014). Según la información sobre permisos otorgados por la Dirección de Aguas del MINAE en el 2017, se concedieron 1749 puntos de vertido de aguas residuales, el $69,6 \%$ de las autorizaciones corresponde a actividades de comercio y servicios, un $17,3 \%$ al sector agropecuario, un $10,6 \%$ a la industria manufacturera y el $2,5 \%$ a otros entes. De este total, el $62 \%$ de los vertidos es de tipo ordinario y un $36 \%$ de tipo especial (HerreraMurillo, 2017).

Los datos anteriores, evidencian que actualmente los recursos hídricos en Costa Rica cumplen una función de recurso económico, en ese sentido, la teoría economía de mercado indica que la oferta y la demanda, determinan la asignación de los recursos a través del sistema de precios, buscando de forma paralela el bienestar humano (Baumol y Oates, 1988; Chopra y Das, 2019). Sin embargo, existen fallas de mercado que provocan una desvalorización de los daños ocasionados al ambiente, producto de la actividad económica; esto se conoce con el nombre de externalidades negativas por lo que la economía ambiental procura crear mercados por medio de impuestos y/o subsidios para los bienes o recursos y de esta forma corregir estas externalidades (Chopra y Das, 2019; Hernández-Sancho et al., 2010).
Actualmente Costa Rica posee instrumentos de regulación y de valoración económica del recurso hídrico con el objetivo de contribuir a la disminución de la contaminación, no obstante, estas políticas y reglamentos no se han desarrollado con base a estudios y monitoreos integrales sobre la calidad actual de los ríos del país (Bower, 2014); en el 2016 inició el plan nacional de monitoreo de la calidad de los cuerpos de agua superficial, con el propósito de clasificar la calidad del agua en las principales cuencas hidrográficas (MINAE, 2017), no obstante el monitoreo a nivel país ha sido escaso y con estudios que no han integrado un enfoque ecosistémico de calidad (Mena-Rivera et al., 2018). En el decreto 34431-MINAE, "Reglamento del Canon Ambiental por Vertidos" (CAV) se regula el pago por verter sustancias contaminantes en cuerpos de agua superficiales. Hipotéticamente este instrumento económico, se diseñó para valorar el servicio ambiental brindado por el río en la remoción de carga contaminante y a su vez el daño causado al ambiente por el vertido de contaminantes al ecosistema.

El objetivo de este trabajo es presentar un análisis del diseño de la metodología de valoración económica empleada en el CAV mediante la identificación del marco legal e institucional de dicho reglamento, en conjunto con la comprensión de las funciones ambientales afectadas por vertidos de aguas residuales a cuerpos receptores. Así como, evidenciar la situación actual en términos económicos de algunos casos de vertidos donde se aplica el canon y finalmente realizar recomendaciones que servirían como insumo de análisis para posibles mejoras en reformas del reglamento.

\section{Metodología}

La investigación realizada es del tipo exploratoria con un enfoque cuantitativo en la que se realizó un análisis metodológico de la formulación del CAV publicado en el decreto 34431-MINAE. Además, se analizó la aplicación de este reglamento en datos obtenidos del Ministerio de Salud sobre vertidos realizados en el río Virilla. A continuación, se describen los principales componentes metodológicos de esta investigación.

\section{Descripción del Reglamento del CAV:}

Se realizó una descripción de los principales aspectos que constituyen el reglamento y que permiten analizar su formulación, entre ellos destacan: base legal, fecha de aprobación, objeto y ámbito de aplicación, naturaleza, fundamento, base para el cobro, monto y uso de los recursos generados. La información anterior fue obtenida del decreto 34431-MINAE. 


\section{Descripción cuantitativa del CAV}

Se describió las fórmulas utilizadas para el cálculo del CAV por parte de las actividades productivas, estas son dependientes del uso del agua y del cumplimiento por parte de la actividad productiva del decreto 33601-S: Reglamento de vertido y reusó de aguas residuales (RVRAR).

Evaluación del diseño de la metodología actual de valoración económica utilizada en el reglamento de CAV

Se determinó los criterios utilizados por el MINAE para fijar los montos actuales de cobro de CAV, lo anterior se realizó mediante entrevista al personal del MINAE llevada a cabo en mayo 2017 (Chinchilla, 2017) y utilizando fuentes bibliográficas disponibles, es importante destacar la escasez de información sobre este tema, siendo uno de los objetivos principales de esta investigación esclarecer estos criterios metodológicos.

Aplicación del CAV a datos reales de Reportes Operacionales

Se consultó una base de datos obtenida del Ministerio de Salud (2014) con los resultados de los reportes operacionales de aguas residuales (ROAR) de diferentes entes generadores cuyos vertidos son dispuestos en el río Virilla. A partir de esta información se logró caracterizar la procedencia e identidad de las actividades comerciales generadoras, con el objetivo de tomar como caso de estudio la actividad de mayor caudal vertido.
Determinación de las funciones ambientales afectadas por el vertido de aguas residuales:

Se realizó un cuadro comparativo para relacionar los principales servicios ambientales de los cuerpos de agua, con sus funciones y afectaciones en caso de contaminación del recurso hídrico. Estos se obtuvieron a través de la búsqueda bibliográfica especializada.

Definición de consideraciones para la mejora de la metodología de valoración económica.

Con el análisis de la información anterior, se realizó una definición de consideraciones para la mejora de la metodológica de valoración económica, en ella se busca la integración del componente del daño ambiental en el cobro del CAV y de la estimación de valores de no uso; para que de esta forma sirva como insumo de mejora y estudio en futuras reformas del reglamento.

\section{Discusión y resultados}

\section{Descripción del Reglamento del CAV}

En el decreto 34431-MINAE se estable que en Costa Rica es permitido verter aguas residuales a cuerpos receptores mediante previo tratamiento y posterior control de la concentración de algunos contaminantes respecto a los límites máximos permisibles por el RVRAR. En el cuadro 1, se realiza una presentación descriptiva del reglamento en estudio, abordando aspectos generales que permitan contextualizar el caso.

Cuadro 1. Matriz de aspectos descriptivos del Decreto No 34431 "Reglamento del CAV".

Aspectos
Base legal
Fecha de aprobación e inicio
Objeto de aplicación

Ámbito y sujeto de aplicación

Naturaleza del Canon

Fundamento

Base para el cobro

Detalle
Decreto Ejecutivo No. 34431 MINAE. (No. 31176 MINAE)
04 de marzo del 2008 (22 de abril 2003)

La regulación del canon por uso del recurso hídrico, para verter sustancias contaminantes

Las personas físicas o jurídicas, públicas o privadas, que utilicen los cuerpos de agua para introducir, transportar, y/o eliminar vertidos, que puedan provocar modificaciones en la calidad física, química o biológica del agua.

Es un instrumento económico de regulación que se fundamenta en el principio de "quien contamina paga" y que pretende el objetivo social de alcanzar un ambiente sano y ecológicamente equilibrado, de conformidad con lo dispuesto en el artículo 50 de la Constitución Política, a través del cobro de una contraprestación en dinero a quienes usen el servicio ambiental de los cuerpos de agua.

El uso directo, o indirecto de los cuerpos de agua para verter en ellos sustancias nocivas que de algún modo alteren y/o generen daños en su calidad, al ambiente o a la sociedad.

Se cobra sobre la carga contaminante neta vertida, medida en kilogramos, de los parámetros de contaminación denominados "Demanda Química de Oxígeno" (DQO) y "Sólidos Suspendidos Totales" (SST). El monto se determina tomando en cuenta los siguientes elementos: 
- El costo equivalente a remover un kilogramo de los parámetros utilizados mediante el uso de la tecnología idónea disponible.

- Los costos de los daños asociados con la contaminación hídrica calculados mediante las técnicas de valoración económica que defina el MINAE

\section{Monto del Canon}

\section{Recursos generados}

Zona de aplicación de cobro

Institución encargada implementación y manejo Aspectos

Base legal

Fecha de aprobación e inicio Objeto de aplicación

Ámbito y sujeto de aplicación

Naturaleza del Canon

Fundamento

Base para el cobro
Es importante mencionar que este último aspecto no ha podido ser calculado y actualmente se está cobrando con base al primer aspecto considerado.

Se cobra la suma de 0,22 dólares (veintidós centavos de dólar), o su equivalente en colones, por cada kilogramo de DQO vertido y de 0,19 dólares (diecinueve centavos de dólar) o su equivalente en colones por cada kilogramo de SST vertido. El tipo de cambio será el vigente al momento de la facturación.

Los recursos generados se asignan de la siguiente manera:

- 60\%: en apoyo al financiamiento a inversiones de proyectos de alcantarillado sanitario y tratamiento de aguas residuales domésticas.

- 15\%: para la promoción de la producción más limpia en fuentes puntuales de vertidos de aquellos sectores contemplados en el artículo 2 del reglamento, principalmente en actividades de capacitación, divulgación e investigación.

- 10\%: para financiar los requerimientos de monitoreo de las fuentes emisoras, incluyendo la identificación de fuentes generadoras de efluentes, la toma de muestras de los vertidos, el análisis de laboratorio, estudios técnicos sobre la calidad del agua en los cuerpos de agua y otros aspectos referidos a la medición, estimación y control de las descargas

- 10\%: para financiar los gastos de administración del canon, incluyendo los requerimientos de registro y bases de datos de fuentes generadoras, cálculo de los montos que cada emisor debe pagar, facturación, gestión de la recaudación y otros gastos de administración.

- 5\%: para actividades de educación ambiental dirigidos a la población y demás usuarios del agua.

Conjunto de cuencas, cuenca, subcuenca o tramos de cuenca delimitados para la aplicación del canon ambiental por vertidos.

de Ministerio de Ambiente y Energía (MINAE)

\section{Detalle}

Decreto Ejecutivo No. 34431 MINAE. (No. 31176 MINAE)

04 de marzo del 2008 (22 de abril 2003)

La regulación del canon por uso del recurso hídrico, para verter sustancias contaminantes

Las personas físicas o jurídicas, públicas o privadas, que utilicen los cuerpos de agua para introducir, transportar, y/o eliminar vertidos, que puedan provocar modificaciones en la calidad física, química o biológica del agua.

Es un instrumento económico de regulación que se fundamenta en el principio de "quien contamina paga" y que pretende el objetivo social de alcanzar un ambiente sano y ecológicamente equilibrado, de conformidad con lo dispuesto en el artículo 50 de la Constitución Política, a través del cobro de una contraprestación en dinero a quienes usen el servicio ambiental de los cuerpos de agua.

El uso directo, o indirecto de los cuerpos de agua para verter en ellos sustancias nocivas que de algún modo alteren y/o generen daños en su calidad, al ambiente o a la sociedad.

Se cobra sobre la carga contaminante neta vertida, medida en kilogramos, de los parámetros de contaminación denominados "Demanda Química de Oxígeno" (DQO) y "Sólidos Suspendidos Totales" (SST). El monto se determina tomando en cuenta los siguientes elementos: 
- El costo equivalente a remover un kilogramo de los parámetros utilizados mediante el uso de la tecnología idónea disponible.

- Los costos de los daños asociados con la contaminación hídrica calculados mediante las técnicas de valoración económica que defina el MINAE

\section{Monto del Canon}

\section{Recursos generados}

\section{Zona de aplicación de cobro}

Institución encargada implementación y manejo
Es importante mencionar que este último aspecto no ha podido ser calculado y actualmente se está cobrando con base al primer aspecto considerado.

Se cobra la suma de 0,22 dólares (veintidós centavos de dólar), o su equivalente en colones, por cada kilogramo de DQO vertido y de 0,19 dólares (diecinueve centavos de dólar) o su equivalente en colones por cada kilogramo de SST vertido. El tipo de cambio será el vigente al momento de la facturación.

Los recursos generados se asignan de la siguiente manera:

- 60\%: en apoyo al financiamiento a inversiones de proyectos de alcantarillado sanitario y tratamiento de aguas residuales domésticas.

- 15\%: para la promoción de la producción más limpia en fuentes puntuales de vertidos de aquellos sectores contemplados en el artículo 2 del reglamento, principalmente en actividades de capacitación, divulgación e investigación.

- 10\%: para financiar los requerimientos de monitoreo de las fuentes emisoras, incluyendo la identificación de fuentes generadoras de efluentes, la toma de muestras de los vertidos, el análisis de laboratorio, estudios técnicos sobre la calidad del agua en los cuerpos de agua y otros aspectos referidos a la medición, estimación y control de las descargas

- 10\%: para financiar los gastos de administración del canon, incluyendo los requerimientos de registro y bases de datos de fuentes generadoras, cálculo de los montos que cada emisor debe pagar, facturación, gestión de la recaudación y otros gastos de administración.

- 5\%: para actividades de educación ambiental dirigidos a la población y demás usuarios del agua.

Conjunto de cuencas, cuenca, subcuenca o tramos de cuenca delimitados para la aplicación del canon ambiental por vertidos.

de Ministerio de Ambiente y Energía (MINAE)
Este tipo de decretos se consideran como instrumentos económicos para la "adecuada gestión del agua"; cuyo principal objetivo es que las actividades económicas que hacen uso de los servicios ambientales de los cuerpos receptores para el procesamiento de las aguas residuales deban pagar al estado por los servicios de depuración de la carga contaminante y la externalidad negativa generada a la sociedad costarricense, basándose en el principio de "el que contamina paga" (Ortega, 2006).

\section{Descripción cuantitativa del CAV}

Como fue descrito en el cuadro 1, para determinar la magnitud de la externalidad negativa por contaminación del agua, el CAV incluye las fórmulas de cálculo con base en 2 parámetros de contaminación: Demanda
Química de Oxígeno soluble (DQO soluble) y Sólidos Suspendidos Totales (SST), lo anterior, se debe a que en la formulación del reglamento se consideró que estos parámetros son representativos del tipo principal de carga contaminante vertida a los cuerpos de agua por parte de los entes generadores, además reflejan los efectos de la carga orgánica contaminante biodegradable y de aquella que requiere de procesos químicos para su oxidación (SST). Aunado a lo anterior son parámetros de fácil monitoreo por métodos convencionales cuyo impacto contaminante en los cuerpos de agua está principalmente asociado con el nivel total de la carga acumulada y no por vertidos puntuales. Finalmente son parámetros que se pueden prevenir mediante procesos de producción más limpia, lográndose a un nivel de costo relativamente más bajo en comparación con otras sustancias contaminantes (López y Méndez, 2004). 
La aplicación del CAV es dependiente del tipo de cálculo según sea el escenario (López, 2003): uso que el generador hace sobre el recurso y el cumplimiento de los parámetros de vertido de acuerdo RVRAR, de lo anterior se derivan tres fórmulas matemáticas para la aplicación en el

a) Los parámetros cumplen lo establecido en el RAVRAR:

$$
\text { Monto }_{j}=\frac{Q \times 0,0864 \times \frac{t}{24} \times\left(C v_{j}-C n_{j}\right) \times M_{j} \times T \times P \times 0,75}{4}(\text { Ecuación 1) }
$$

b) Los parámetros no cumplen lo establecido en el RAVRAR:

$$
\text { Monto }_{j}=\frac{Q \times 0,0864 \times \frac{t}{24} \times M_{j} \times T \times P \times\left[\left(C p_{j}-C a_{j}\right)+\left(3,5 \times\left(C v_{j}-C p_{j}\right)\right)\right]}{4}(\text { Ecuación 2) }
$$

c) El ente generador hace uso no consuntivo del agua:

$$
\text { Monto }_{j}=\frac{Q \times 0,0864 \times \frac{t}{24} \times\left(C v_{j}-C n_{j}\right) \times M_{j} \times T \times P \times K}{4} \quad(\text { Ecuación 3) }
$$

Donde:

j: Parámetro de contaminación objeto de cobro.

Monto j: Monto a cobrar por trimestre, por concepto del canon ambiental por vertidos del parámetro j, en colones.

Q: Caudal promedio vertido del usuario, expresado en litros por segundo (L/s).

0,0864: Factor de conversión para las magnitudes de tiempo y masa.

$\mathrm{t}$ : Tiempo promedio de vertido al día del usuario, expresado en horas.

0,75: Factor de descuento por cumplimiento del RAVRAS

$\mathrm{Mj}$ : Monto correspondiente al parámetro j, en colones por kilogramo (colones $/ \mathrm{kg}$ ).

Evaluación del diseño de la metodología de valoración económica utilizada en el reglamento de CAV

El método de valoración utilizado para la determinación del valor económico de la descarga de aguas residuales en cuerpos receptores tomó como base un estudio realizado en la cuenca del Río Grande de Tárcoles para el Proyecto de Alcantarillado Sanitario de la GAM, realizado por el por el Instituto Costarricense de Acueductos y Alcantarillados (ICAA) entre los años 1997-1998.

Los factores involucrados para la definición de estos montos específicamente se encuentran relacionados a los costos de inversión requeridos para terrenos, construcción de instalaciones, sistemas de tratamiento, redes de colección, sistemas de bombeo, costos anuales de operación y mantenimiento (COM); así como los costos unitarios de remoción para los contaminantes base de cobro y su respectiva carga anual estimada. Además, para obtener estos valores, los formuladores del CAV tomaron como base una población servida para el 2025 de 1.632 .937 personas, generado $180 \mathrm{~L} /$ día de agua residual con una carga en DQO de 667 mg/L y 500 mg/L de S.S.T.

En el cuadro 2 se muestran los valores obtenidos por el estudio técnico y financiero, lo cuales fueron la base de estimación para los montos fijados en el CAV. Estos valores reflejan el costo de evitar la contaminación (costo de mitigación o de abatimiento), pero no incorporan el costo monetario del daño asociado con esa contaminación, por lo que se trata de un valor que subestima el verdadero costo que implican las externalidades negativas provocadas por los entes generadores. Esto, a pesar de que el decreto en su constitución proponía estimar los costos de los daños asociados con la contaminación hídrica, calculados mediante las técnicas de valoración económica que 
defina el MINAE.

Cuadro 2. Costo por unidad de carga removida en dólares para el cobro de CAV. (López y Méndez, 2004)

\begin{tabular}{cc}
\hline Concepto & Valor \\
\hline $\begin{array}{c}\text { Costo Anual equivalente } \\
\text { de remoción DQO }(\$)\end{array}$ & $24,454,058.04$ \\
\hline $\begin{array}{c}\text { Costo Anual equivalente } \\
\text { de remoción SST }(\$)\end{array}$ & $18,278,100.40$ \\
\hline $\begin{array}{c}\text { Cantidad anual de DQO } \\
\text { removida }(\mathrm{Kg})\end{array}$ & $113,490,931.51$ \\
\hline $\begin{array}{c}\text { Cantidad anual de SST } \\
\text { removida }(\mathrm{Kg})\end{array}$ & 0,22 \\
\hline $\begin{array}{c}\text { Costo unitario de } \\
\text { remoción DQO }(\$ / \mathrm{Kg})\end{array}$ \\
\hline $\begin{array}{c}\text { Costo unitario de } \\
\text { remoción SST }(\$ / \mathrm{Kg})\end{array}$ \\
\hline $\begin{array}{c}\text { Costo anual equivalente }=\text { costos anuales de capital }+ \\
\text { costos anuales de COM }\end{array}$ \\
\hline $\begin{array}{c}\text { Datos de proyección al } 2025 \text { con la mayor capacidad } \\
\text { instalada del sistema }\end{array}$ \\
\hline
\end{tabular}

Aplicación del CAV a datos reales de Reportes Operacionales

Se consultó una base de ROAR suministrada por el Ministerio de Salud (2014), en la cual se sistematizaba datos relacionados a los vertidos realizados en el río Virilla durante el periodo comprendido del IC2012IIC2014. La caracterización de los vertidos por código CIIU expresado como carga contaminante (Caudal por DQO), se muestra en la figura 1, destacando que a pesar de existir más de 10 tipos de actividades productivas con permiso de vertido al río Virilla, el $82,3 \%$ del total de la carga es aportado por actividad con el código CIIU 7010, relativa a la actividad de alcantarillado sanitario. Esta distribución se explica debido a que esta actividad posee caudales diarios de vertido de $3138 \mathrm{~m}^{3} /$ día, por esta razón se decide hacer una revisión de los reportes operacionales del ente generador con código CIIU 7010, que mayor cantidad de reportes consigne en la base de datos, con el objetivo de conocer los montos pagados por el mismo bajo el escenario del actual reglamento.

Para el caso de estudio desarrollado, se aplicaron las ecuaciones 1 y 2 ya que no existe uso no consuntivo y además existe incumplimiento en ambos parámetros. La concentración de DQO y SST del sitio de estudio se estimó a partir de los resultados publicados por MenaRivera y otros (2018), donde se evaluó la calidad del agua superficial del río Virilla del 2014-2016, además, al tratarse de alcantarillado sanitario, se asumió que el ente generador vierte al menos durante 30 días del mes en un periodo de 8 horas. En el cuadro 3 se muestran las estimaciones de los montos que debió pagar el ente generador en cada periodo respectivo.

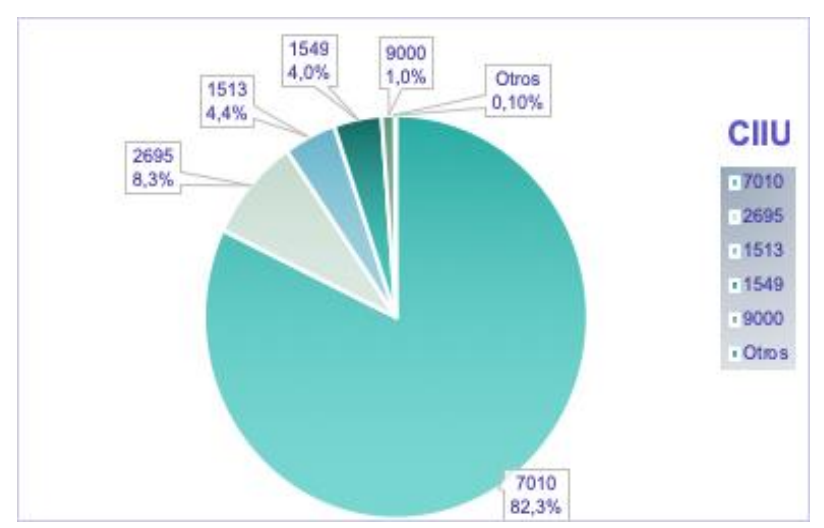

Figura 1. Porcentaje de carga orgánica vertida por día el río Virilla según el código CIIU del ente generador.

Cuadro 3. Aplicación del Decreto Ejecutivo No. 34431 MINAE Reglamento de Canon por Vertidos

\begin{tabular}{|c|c|c|c|c|c|}
\hline Año & $\begin{array}{c}\text { Caudal } \\
(\mathrm{L} / \mathrm{s})\end{array}$ & $\begin{array}{c}\text { DQO } \\
(\mathrm{mg} / \mathrm{L})\end{array}$ & $\begin{array}{c}\text { SST } \\
(\mathrm{mg} / \mathrm{L})\end{array}$ & $\begin{array}{c}\text { Monto } \\
\text { por DQO } \\
(\$)\end{array}$ & $\begin{array}{c}\text { Monto } \\
\text { por } \\
\text { SST }(\$)\end{array}$ \\
\hline 2012-IC & 35 & 133 & 31 & 1769 & 239 \\
\hline 2012-IIC & 33 & 118 & 36 & 1452 & 287 \\
\hline 2012-IIIC & 37 & 26 & 42 & 161 & 398 \\
\hline 2013-IC & 37 & 366 & 58 & 18734 & 772 \\
\hline 2013-IIC & 37 & 204 & 42 & 6747 & 397 \\
\hline 2013-IIIC & 37 & 184 & 56 & 5291 & 718 \\
\hline 2014-IC & 37 & 191 & 85 & 5896 & 2125 \\
\hline 2014-IIC & 38 & 91 & 7 & 1206 & -143 \\
\hline \multicolumn{4}{|c|}{ Total } & $\$ 41246$ & $\$ 4934$ \\
\hline
\end{tabular}

Desafortunadamente, para alcances de esta investigación, no fue posible la obtención mediante fuente primaria, la cantidad de personas a la que sirvió este generador. Sin embargo, de acuerdo con la ecuación 4 , es posible estimar el número promedio de personas abastecidas por este generador, utilizando como factor de generación de aguas residuales un valor de 180 litros por persona por día (Ruiz, 2012), se logra obtener un valor promedio aproximado de 17684 personas abastecidas por día.

(Ecuación 4) 
Con este valor y en conjunto con los datos del cuadro 3 , puede estimarse el monto total de pago por cada persona en su recibo mensual, tomando como base los resultados obtenidos se estima que una persona mensualmente pagó durante este periodo un total de $\$ 0,082 / \mathrm{mes}$.

Estudios realizados en Grecia, han mostrado que las personas han incrementado su disposición a pagar por servicios de saneamiento de aguas residuales (WTP, "Willing to pay"), puesto que Kontogianni et al (2003) encontraron un WTP del 69\% en la población de estudio, por su contraparte, Tziakisa et al (2009) encontraron un WTP aproximado de 93\%. Estudios más recientes realizados en Malasia por Munusami et al (2016), determinaron un WTP del $87 \%$. Los resultados encontrados por estos autores evidencian una tendencia al cambio hacia una mayor conciencia ambiental, por lo que la realización de un estudio de este tipo en nuestro país a través de métodos de contingencia sería importante para fundamentar futuros cambios a los montos actuales del CAV.
Casos más específicos son evidenciados por estudios de Acey et al (2019) en Kenia, Ezebilo (2013) en Nigeria y Tudela-Mamani et al (2017) en Perú, ya que determinaron WTP's de 2,9 - 2 y 1,46 (\$/mes), respectivamente, como aumento en la facturación mensual de los servicios de aguas con el objetivo de invertir en nuevos sistemas de tratamiento de aguas residuales o mejoramiento de estos. Los valores mencionados anteriormente, sugieren que los precios pagados en Costa Rica únicamente por la remoción de la carga contaminante son bastante bajos.

Determinación de las funciones ambientales afectadas por el vertido de aguas residuales

El deterioro evidente del recurso hídrico por vertidos de tipo especial y/o ordinario a cuerpos de agua, genera que las funciones ambientales del agua se vean afectadas tal y como se describe en el cuadro 4.

Cuadro 4. Funciones ambientales afectadas por la contaminación del recurso hídrico. (Calvo, 2014; López, 2003)

\begin{tabular}{|c|c|c|}
\hline Servicios & \multirow{2}{*}{$\begin{array}{l}\text { Funciones } \\
\text {-Alimento (agricultura y ganadería) } \\
\text { - Procesos bioquímicos } \\
\text { - Agua dulce }\end{array}$} & Afectación \\
\hline $\begin{array}{l}\text { De } \\
\text { aprovisionamiento }\end{array}$ & & $\begin{array}{l}\text { Disminución de la cantidad de agua potable } \\
\text { esencial para el funcionamiento bioquímico } \\
\text { del ser vivo }\end{array}$ \\
\hline Reguladores & $\begin{array}{l}\text { - Reproducción vegetal } \\
\text { - Polinización } \\
\text { - Diseminación de semillas } \\
\text { - Regulación de plagas } \\
\text { - Protección contra riesgos naturales } \\
\text { - Regulación de la erosión } \\
\text { - Purificación de aguas }\end{array}$ & $\begin{array}{l}\text { Mayor incidencia de inundaciones por } \\
\text { desbordamiento de ríos, disminución de la } \\
\text { tasa de purificación del agua, enfermedades. }\end{array}$ \\
\hline Culturales & $\begin{array}{l}\text { - Educación y generación de conocimientos } \\
\text { - Recreación y valores estéticos }\end{array}$ & Afectación del paisaje, descontento social \\
\hline De apoyo & $\begin{array}{l}\text { - Producción } \\
\text { primaria } \\
\text { - } \\
\text { Asestrucción de ecosiste } \\
\text { del hábitat } \\
\text {-Ciclo de oxígeno, } \\
\text { nutrientes de } \\
\text { - Formación y } \\
\text { retención del } \\
\text { suelo } \\
\text { - Producción de } \\
\text { oxígeno } \\
\text { atmosférico } \\
\text { - Ciclo del agua } \\
\text { (de los recursos } \\
\text { hídricos) }\end{array}$ & $\begin{array}{l}\text { as acuáticos, disminución de la tasa de } \\
\text { ctación del ciclo hidrológico. }\end{array}$ \\
\hline
\end{tabular}

Consideraciones para la mejora de la metodología de valoración económica del CAV

El CAV desde el punto de vista conceptual según lo expuesto en el cuadro 1, fundamenta su valoración económica en dos grandes aspectos: el costo de remoción de la carga contaminante vertida y los costos asociados a la contaminación hídrica. No obstante, en la evaluación del diseño de la metodología, se evidencia que actualmente solo se contemplan el costo de remoción. La metodología actual empleada del CAV, es un método basado en el valor de uso del recurso hídrico debido a la 
función ambiental de servicio de tratamiento o depuración del agua residual. Específicamente podríamos relacionar esta metodología con un enfoque de valoración directa de mercado por costos de reemplazo, mediante un análisis económico de inversión inicial y costos de mantenimiento de un sistema de tratamiento convencional, estableciéndose el costo unitario por $\mathrm{kg}$ de contaminante vertido.

Lo anterior nos indica que, hoy en día el MINAE no ha podido establecer una metodología de valoración para los costos de los daños asociados con la contaminación hídrica. Esto, debido a que durante muchos años no ha existido la articulación necesaria entre distintos actores importantes de nuestra sociedad, que puedan colaborar con esta institución en la propuesta de metodologías para valorar los daños ocasionados por el vertido de aguas residuales a los cuerpos receptores y que con esto el generador internalice las externalidades negativas, que impactan a la sociedad costarricense (Ministerio del Ambiente y Energía, 2003).

Inicialmente, se recomienda considerar las funciones ambientales expuestas en el cuadro 4, ya que tienen una afectación importante por la contaminación del recurso hídrico. Para la valoración de estos daños sería importante definir una prioridad para tomar en cuenta en el segundo aspecto de valoración del CAV. Esta prioridad debería estar sustentada en una matriz de evaluación de funciones ambientales prioritarias $\mathrm{y}$ significativas en las principales cuencas hidrográficas del país, con el objetivo de que el segundo factor a integrar al cobro integre de una manera holística los diferentes valores de no uso del recurso hídrico en el país.

Posterior a la priorización de las funciones ambientales, se deberían determinar las metodologías de valoración económica aplicables. No obstante, nos parece importante destacar que un componente imperativo del valor económico a evaluar sería el de no uso, esto mediante un método de valoración contingente, que permita conocer cuanto representa para las comunidades cercanas de los principales puntos de vertido, el restaurar y/o conservar la calidad del agua en el cuerpo receptor. En la figura 2 se muestra un diagrama de flujo propuesto para la evaluación de la metodología actual del CAV.

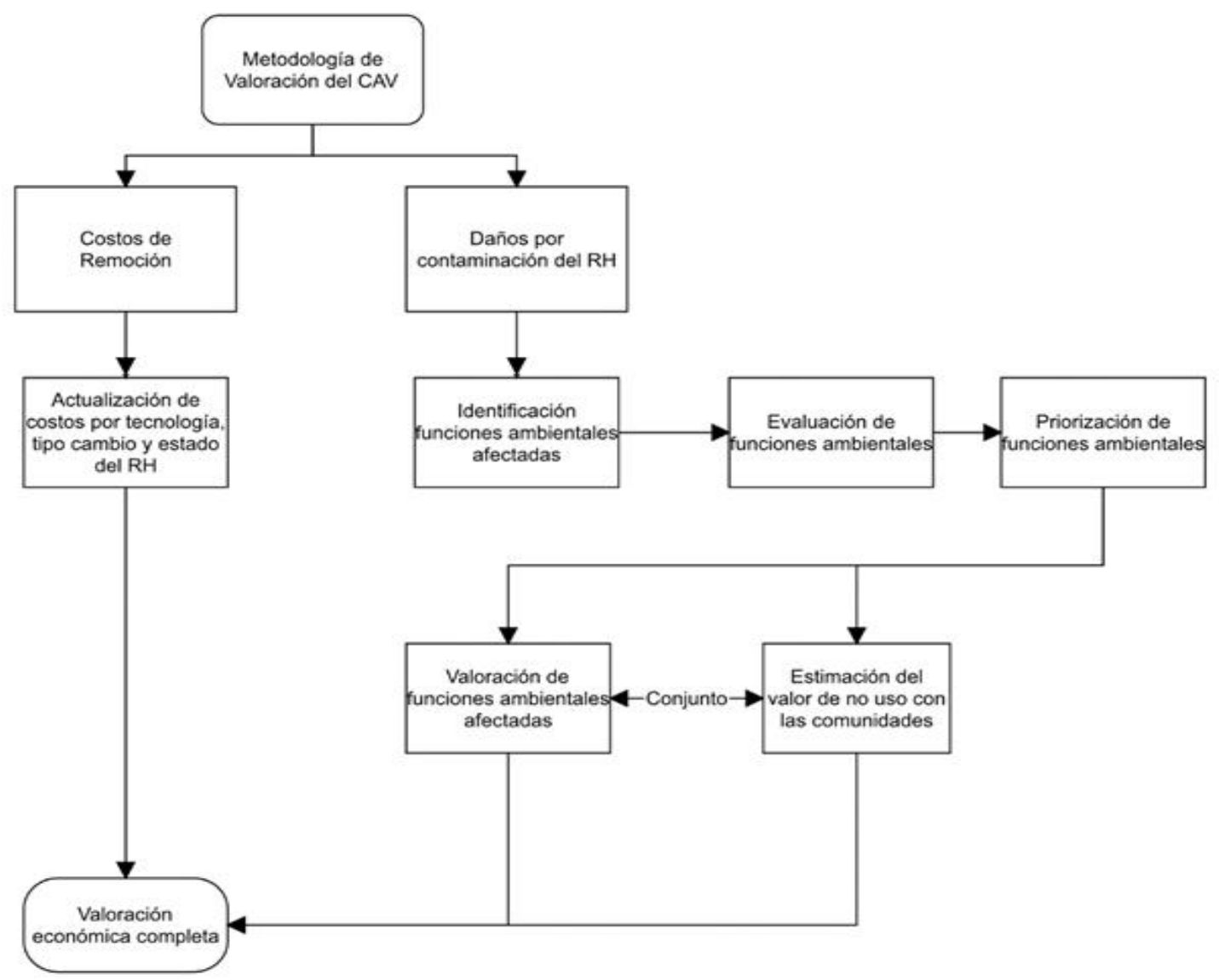

Figura 2. Diagrama de flujo para la propuesta de mejora a la metodología de valoración económica del CAV.

Fuente: Elaboración propia. 


\section{Conclusiones}

El CAV constituye uno de los primeros instrumentos de valoración económica de servicios ambientales existentes en Costa Rica. Su importancia es alta debido a que su constitución pretendía restaurar las condiciones de la calidad de los principales ríos del país, además de generar un proceso de conciencia en la sociedad costarricense. Actualmente este instrumento permite generar fondos que sustenten proyectos de inversión en el área de saneamiento ambiental, permitiendo transformar la internalización de las externalidades en posibles soluciones de la degradación ambiental de nuestros ecosistemas hídricos. No obstante, un hallazgo importante de esta investigación reside en que actualmente el CAV está ejecutándose de manera incompleta según su base de constitución, ya que la metodología mediante la cual se obtuvieron los montos de cobro para cada parámetro solo contempla los valores de uso directo, dejando de lado la estimación de daños ambientales por el uso del recurso hídrico como sistema depurador.

Para la adecuada valoración de los servicios ambientales brindados por los cuerpos receptores, se debe incluir los daños ocasionados al recurso hídrico y como estos repercuten en la sociedad costarricense, el MINAE debe articular esfuerzos con otras instituciones del estado y la academia, con el fin de estimar de manera adecuada los daños que representa la disminución de calidad de los cuerpos de agua en el país. Para esta valoración, deben considerarse (más no limitarse) a las funciones ambientales propuestas en esta investigación, definiendo una priorización en un proceso sistemático donde se interrelaciona cada función a una metodología de estimación adecuada. Por ejemplo, aquellos valores de no uso deben estimarse a través de una valoración no contingente, integrando a su vez y en la medida de lo posible la participación de diversos actores clave (comunidad, gobiernos locales, actividades productivas, entre otros), con el objetivo de desarrollar esta mejora en el marco de la gestión integrada del recurso hídrico.

El reglamento debería tener procesos de actualización periódicos con el fin de compensar los cambios de tecnologías de tratamiento, tipos de cambio de moneda e inclusive poder contemplar las mejoras o los detrimentos en los que ha incurrido el cuerpo de agua receptor por prestar el servicio, de esta manera se internalizarían las externalidades positivas y/o negativas según corresponda. Desde la entrada en vigor del CAV, el valor de la variable $\mathrm{Mj}$, de las ecuaciones anteriores, ha sido tema de discusión en diversos ámbitos de la gestión de aguas residuales, principalmente debido a dos factores: la crítica hacia el monto relativamente bajo perceptible por las personas y el desconocimiento de los criterios utilizados para la fijación de dichos valores. En esta investigación se logró evidenciar que los montos actuales son bajos si se compara con la disposición de pago para el saneamiento de aguas residuales en otros países. Finalmente, es importante repensar si el método de cálculo basado en dos variables es el idóneo para el cobro del CAV, ya que podría plantearse el utilizar un modelo multivariado que integre las 8 variables de control básico establecidas por el RAVRAR o al menos las que un análisis estadístico robusto revelen que son significativas y correlacionadas con el deterioramiento en la calidad del agua.

\section{Agradecimientos}

Se agradece a el Consejo de Becas de la Universidad Estatal a Distancia y al Fondo de Incentivos del MICIIT por el financiamiento brindado a los autores de este documento en sus estudios de Maestría en Gestión y Estudios Ambientales ya que la producción de este artículo nace en el marco del programa de estudios de esta maestría.

\section{Referencias}

Acey, C., Kisiangani, J., Ronoh, P., Delaire, C., Makena, E., Norman, G. \& Peletz, R. (2019). Crosssubsidies for improved sanitation in low income settlements: Assessing the willingness to pay of water utility customers in Kenyan cities. World Development, 115, 160-177. https://doi.org/10.1016/j.worlddev.2018.11.006

Barrenha, P. I. I., Tanaka, M. O., Hanai, F. Y., Pantano, G., Moraes, G. H., Xavier, C., y Mozeto, A. A. (2018). Multivariate analyses of the effect of an urban wastewater treatment plant on spatial and temporal variation of water quality and nutrient distribution of a tropical mid-order river. Environmental Monitoring and Assessment, 190(1). https://doi.org/10.1007/s10661-017$\underline{6386-4}$

Ballestero, M., y Reyes, V. (2006). Water Quality Managment in Central America: Case Study of Costa Rica. En B. Braga, M. Porto, y L. Meneses (Eds.), Water Quality Management in the Americas (pp. 179-198). New York: Springer Berlin Heidelberg. https://doi.org/10.1007/3-540$\underline{30444-4 \_5}$

Baumol, W. y Oates, W. (1988). The Theory of Environmental Policy. Cambridge: University Press. Retrieved from https://www.cambridge.org/core/books/theory 
-of-environmental-

policy/E8FE3C8AB6D8D6982E5B65CC95F

$\underline{\mathrm{A} 1478}$

Bower, K. M. (2014). Water supply and sanitation of Costa Rica. Environmental Earth Sciences, $71(1)$, $107-123$ https://doi.org/10.1007/s12665-013-2416-x

Calvo, A. (2014). Manual para docentes: Conservación y uso sostenible del servicio ecosistémico del agua. Instituto Costarricense de Acueductos y Alcantarillados (AyA). Retrieved from https://www.aya.go.cr/interactivo/materialDoc entes/El\%20Agua.pdf

Chinchilla, M. (2017). Dirección de Gestión de Calidad Ambiental (DIGECA). Ministerio de Ambiente y Energía. Entrevista por correo electrónico.

Chopra, V., \& Das, S. (2019). Estimating Willingness to Pay for Wastewater Treatment in New Delhi: Contingent Valuation Approach. Ecology, Economy and Society-the INSEE Journal, 2(2), 75-108.

da Rocha, M. P., Dourado, P. L. R., Cardoso, C. A. L., Cândido, L. S., Pereira, J. G., de Oliveira, K. M. P., y Grisolia, A. B. (2018). Tools for monitoring aquatic environments to identify anthropic effects. Environmental Monitoring and Assessment, 190(2). $\quad$ https://doi.org/10.1007/s10661-017$\underline{6440-2}$

Echeverría, J. Cantillo, B. (2013). Instrumentos Económicos para la Gestión del agua. Revista de Ciencias Ambientales, 45, 13-22. https://doi.org/10.15359/rca.45-1.2

Ezebilo, E. E. (2013). Willingness to pay for improved residential waste management in a developing country. International Journal of Environmental Science and Technology, 10(3), 413-422. https://doi.org/10.1007/s13762-012-0171-2

Ha, P. T. T., Kokutse, N., Duchesne, S., Villeneuve, J. P., Bélanger, A., Hien, H. N., y Bach, D. N. (2017). Assessing and selecting interventions for river water quality improvement within the context of population growth and urbanization: a case study of the Cau River basin in Vietnam. Environment, Development and Sustainability,
19(5),

$1701-1729$

https://doi.org/10.1007/s10668-016-9822-7

Hernández-Sancho, F., Molinos-Senante, M., \& SalaGarrido, R. (2010). Economic valuation of environmental benefits from wastewater treatment processes: An empirical approach for Spain. Science of the Total Environment, 408(4), 953957.

https://doi.org/10.1016/j.scitotenv.2009.10.028

Herrera-Murillo, J. (2017). Uso y estado de los recursos: Recurso Hídrico. En Informe Estado de la Nación en Desarrollo Sostenible 2017 (pp. 331). San José, Costa Rica: Programa Estado de la Nación. Retrieved from http://estadonacion.or.cr/files/biblioteca virtu al/023/Ambientales/Corrales_L_2017a.pdf

Kontogianni, A., I. H. Langford, A. Papandreou, and M. S. Skourtos. (2003). —-Social Preferences for Improving Water Quality: An Economic Analysis of Benefits from Wastewater Treatment Water Resources Management 17 (5): 317-336 https://doi.org/10.1023/A:1025866818391

López, R. Méndez, H. (2004). Un canon por vertidos para el control de la contaminación hídrica. Comisión Centroamericana de Ambiente y Desarrollo (CCAD).

López, R. (2003). “Canon ambiental por vertidos”. Un instrumento económico alternativo para el control y la prevención de la contaminación hídrica en Costa Rica: Instructivo para el cálculo del monto a pagar.

Massoud, M. A. (2012). Assessment of water quality along a recreational section of the Damour River in Lebanon using the water quality index. Environmental Monitoring and Assessment, 184(7), 4151-4160. https://doi.org/10.1007/s10661-011-2251-z

Mena-Rivera, L., Vásquez-Bolaños, O., Gómez-Castro, C., Fónseca-Sánchez, A., Rodríguez-Rodríguez, A., \& Sánchez-Gutiérrez, R. (2018). Ecosystemic Assessment of Surface Water Quality in the Virilla River : Towards Sanitation Processes in. Water, $10,1-16$. https://doi.org/10.3390/w10070845

Ministerio de Ambiente y Energía, Ministerio de Salud \& Instituto Costarricense de Acueductos y 
Alcantarillado. (2016). Política Nacional de Saneamiento en Aguas Residuales. 72-92

Ministerio del Ambiente y Energía de Costa Rica, Programa de Modernización de los Sistemas de Gestión Ambiental en Centroamérica (PROSIGA), Comisión Centroamericana de Ambiente y Desarrollo (CCAD). (2003). Canon Ambiental por Vertidos: Un instrumento económico alternativo para el control y la prevención de la contaminación hídrica en Costa Rica.

Munusami, C., Othman, J., Ismail, S. M., \& Siwar, C. (2016). Estimation of willingness to pay for wastewater treatment service improvement in Malaysia. International Journal of Business and Society, $\quad$ 17(2), 365-374. https://doi.org/10.33736/ijbs.530.2016

Onestini, M. (2011). Water quality and health in poor urban areas of Latin America. International Journal of Water Resources Development, 27(1), 219-226.

https://doi.org/10.1080/07900627.2010.537244

Ortega, L. (2006). Los Instrumentos económicos en la gestión del agua. El caso de Costa Rica. Unidad de Recursos y Energía, CEPAL, 19-29. Retrieved from http://biblioteca.ues.edu.sv/revistas/10800059. pdf

Poltronieri, F.; Cabrera, J. (2016). Impacto Ambiental y Mitigación de Daños, Primer edición.; EUNED: Costa Rica, 2016; pp:1713-257.

Ruiz, F. (2012). Gestión de las Excretas y Aguas Residuales en Costa Rica. Instituto Costarricense de Acueductos y Alcantarillados. Recuperado de https://www.aya.go.cr/centroDocumetacion/catal ogoGeneral/Gestión de las Excretas y Aguas Residuales en Costa Rica Situación Actual y Perspectiva.pdf

Tudela-Mamani, J. W. (2017). Disponibilidad a pagar por el mejoramiento en el tratamiento de aguas residuales: Aplicación del método de valoración contingente en Puno, Perú. Revista Chapingo, Serie Ciencias Forestales y Del Ambiente, 23(3), 191-213.

https://doi.org/10.5154/r.rchscfa.2016.11.059
Tziakisa, I., I. Pachiadakisa, M. Moraitakisa, K. Xideasa, G. Theologisa, and K. P. Tsagarakis. (2009). -Valuing benefits from wastewater treatment and reuse using contingent valuation methodology. Desalination 237: 117-125. https://doi.org/10.1016/j.desal.2007.12.028 